Examining microstructural evolution of Portland cements by in-situ synchrotron micro-tomography

Matteo Parisatto, Maria Chiara Dalconi, Luca Valentini, Gilberto Artioli, Alexander Rack, Rémi Tucoulou, Giuseppe Cruciani, et al.

\section{Journal of Materials Science}

Full Set - Includes 'Journal of Materials Science Letters'

ISSN 0022-2461

J Mater Sci

DOI 10.1007/s10853-014-8743-9

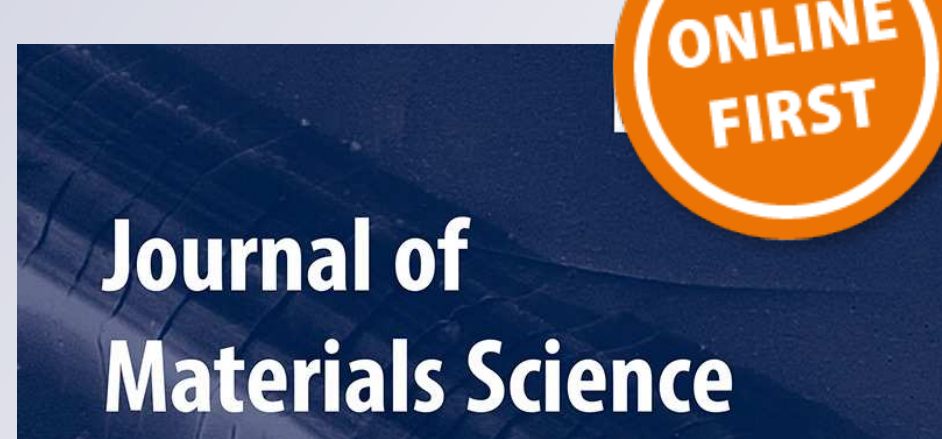

Q) Springer jMs

算 Springer 
Your article is protected by copyright and all rights are held exclusively by Springer Science +Business Media New York. This e-offprint is for personal use only and shall not be selfarchived in electronic repositories. If you wish to self-archive your article, please use the accepted manuscript version for posting on your own website. You may further deposit the accepted manuscript version in any repository, provided it is only made publicly available 12 months after official publication or later and provided acknowledgement is given to the original source of publication and a link is inserted to the published article on Springer's website. The link must be accompanied by the following text: "The final publication is available at link.springer.com". 


\title{
Examining microstructural evolution of Portland cements by in-situ synchrotron micro-tomography
}

\author{
Matteo Parisatto • Maria Chiara Dalconi - Luca Valentini • \\ Gilberto Artioli • Alexander Rack • Rémi Tucoulou • \\ Giuseppe Cruciani • Giorgio Ferrari
}

Received: 12 September 2014/ Accepted: 24 November 2014

(C) Springer Science+Business Media New York 2014

\begin{abstract}
The application of synchrotron radiation X-ray computed micro-tomography (SR $\mathrm{X}-\mu \mathrm{CT}$ ) as a non-invasive approach to the microstructural investigation of Portland cement binders during hydration is presented. The two- and three-dimensional $\mu \mathrm{m}$-scale imaging of undisturbed samples at hydration ages from $\sim 1.5 \mathrm{~h}$ to 3 days is used to obtain a direct visualization of the spatial and temporal relationships between different cement paste components. The microstructural evolution of two cementitious systems during the early stages of hydration is successfully monitored from the comparison of tomographic slices and volumes, clearly showing the progressive growth of hydration phases; the changes in the amount of porosity and unreacted clinker are also quantified. Some critical issues related to the experimental setup and data processing are addressed and discussed as well. Furthermore, a simple procedure to estimate the mean X-ray absorption coefficient of cement pastes from X-ray radiographs is illustrated. The results confirm the potentialities of synchrotron-based X-ray computed micro-tomography
\end{abstract}

\author{
M. Parisatto $(\varangle) \cdot$ M. C. Dalconi · L. Valentini · G. Artioli \\ Department of Geosciences, Università degli Studi di Padova, \\ 6 Via G. Gradenigo, 35131 Padua, Italy \\ e-mail: matteo.parisatto@gmail.com \\ M. C. Dalconi \\ e-mail: mariachiara.dalconi@unipd.it \\ L. Valentini \\ e-mail: luca.valentini@unipd.it \\ G. Artioli \\ e-mail: gilberto.artioli@unipd.it
}

M. C. Dalconi · L. Valentini - G. Artioli

Centro Interdipartimentale di Ricerca per lo Studio dei Materiali Cementizi e dei Leganti Idraulici (CIRCe), 6 Via G. Gradenigo, 35131 Padua, Italy for the three-dimensional investigation of $\mu \mathrm{m}$-scale modifications in hydrating cement pastes with an adequate time resolution, thus providing a real in-situ monitoring of the microstructural evolution of such complex materials.

\section{Introduction}

Understanding the microstructural evolution and the chemical reactions taking place in cement-based materials during their setting and hardening is one of the most challenging goals of modern materials science. It is widely acknowledged that the performance and durability of cements and concretes are controlled by their microstructure, specifically by the pore network properties and the spatial relationships between various hydration products. Among the most widely employed experimental techniques for the investigation of the microstructural features in cementitious systems, scanning electron microscopy (SEM) has played a major role in the past and still remains

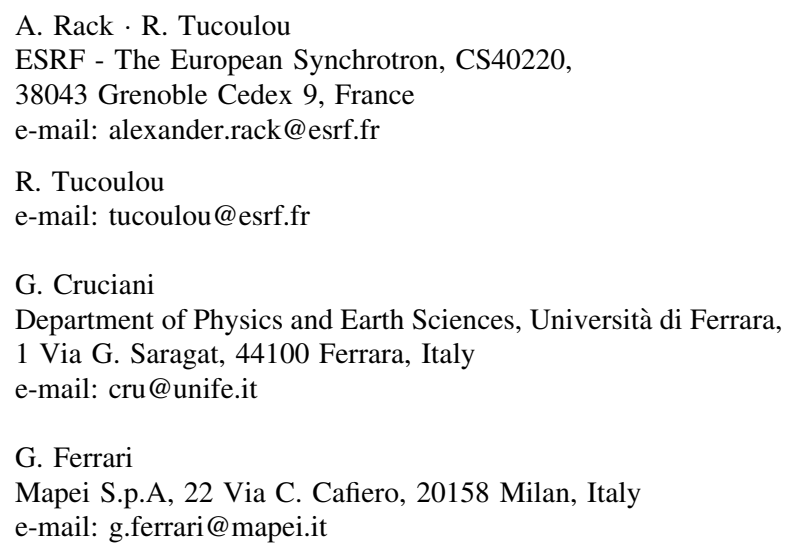


a fundamental tool in this research field. The combination of 2D backscattered electron (BSE) imaging with X-ray energy dispersive spectroscopy (EDS) on polished surfaces allows easy identification and quantification of both clinker phases and hydration products [1, 2]. In addition, excellent morphological information can be obtained using secondary electron (SE) imaging on fresh fracture surfaces [3]. However, the lack of access to real three-dimensional (3D) information represents one of the major limitations of SEM-based techniques when trying to extract quantitative information, e.g. on pore connectivity and other microstructural properties. Moreover, the sample preparation for electron microscopy is often very invasive, not only due to sample cutting and polishing, but also to the exposure to high vacuum conditions inside the SEM chamber that may induce modifications in the microstructure of the hydrating paste. This latter aspect has been mostly solved after the advent of the environmental SEM (ESEM) technology but sample cutting and polishing still remain two critical issues. In particular, polishing poses serious problems in early age samples that have not attained a sufficient hardening. For these reasons, during the last decade, efforts have been devoted to the developing of non-invasive imaging methods for the investigation of cement-based materials.

Nowadays, X-ray computed micro-tomography (X$\mu \mathrm{CT}$ ), which represents the high resolution adaptation of the computed axial tomography technique (CAT) employed in medical applications, offers the great advantage of visualizing the internal microstructure of undisturbed samples in a three-dimensional manner. X-ray computed micro-tomography [4] is an imaging technique based on the virtual reconstruction of the inner mass distribution of an object from 2D X-ray radiographs (or projections) collected at different viewing angles, while the sample rotates relative to a fixed source-detector pair. The final result of the reconstruction process is a series of crosssectional images called slices that, when stacked together, provide a full 3D map of the spatial variations of the X-ray linear attenuation coefficient $(\mu)$ within the investigated object. Each slice is formed by a matrix of voxels (volume elements) whose grey values are proportional to the mean value of $\mu$ in the corresponding volume of the real sample; generally, dark is associated with low- and bright with high-absorbing materials. The maximum spatial resolution achievable can reach the sub-micrometre scale for standard applications and is related not only to the technical specifications of the experimental setup (i.e. X-ray source and detector), but also to the size of the sample. As a general rule, higher resolutions require smaller samples due to the limited field of view of X-ray area detectors.

The investigation of the micro- and nano-scale details of the complex microstructure of cement-based materials necessarily requires the most advanced $\mathrm{X}-\mu \mathrm{CT}$ setups, which can be achieved in synchrotron radiation (SR) facilities. Besides a better spatial resolution, several advantages are offered by SR X- $\mu \mathrm{CT}$ compared to conventional laboratory (cone beam) systems, including faster acquisition times, a more accurate reconstruction process (owing to the parallel beam geometry), increased sensitivity/contrast, and a general minimization of image artefacts. With the improved capabilities of X-ray microtomography systems in SR facilities, it has now become possible to investigate the three-dimensional microstructure of mm-sized cementitious samples with a spatial resolution up to $1 \mu \mathrm{m}$. In the last decade, SR X- $\mu$ CT has been successfully applied to the study of cement binders and other building materials for the extraction of qualitative and quantitative 3D information about pore structure properties, phase evolution, particles shape and other features of interest [5-12]. Furthermore, in the last few years, other synchrotron-based experimental tools related to $\mathrm{X}-\mu \mathrm{CT}$, with enormous potential applications for the cement community, have become available. As an example, the combination of pencil-beam $\mathrm{X}-\mu \mathrm{CT}$ with spatially resolved powder diffraction has led to the development of diffraction tomography (XRD-CT) methods [13-17]. In XRD-CT, phases are identified on the basis of crystallographic information (inter-planar spacings) instead of $\mathrm{X}$-ray attenuation, allowing a direct spatial identification and mapping of many cement phases (including calciumsilicate hydrates, $\mathrm{C}-\mathrm{S}-\mathrm{H}$ ) that could be hardly differentiated from each other using conventional $\mathrm{X}-\mu \mathrm{CT}$, owing to the very limited contrast between their attenuation coefficients.

In the present work, SR X- $\mu \mathrm{CT}$ has been used to visualize and quantify the phase evolution of hydrating Portland cement pastes during the initial stages of hydration, in order to perform a real-time in-situ monitoring of the microstructural modifications occurring during setting and hardening and assess the capabilities and limitations of the technique in time-resolved applications. Two Portland cement pastes prepared with standard formulations were selected for this study and analysed at increasing hydration ages, from approximately $2 \mathrm{~h}$ to 3 days. Moreover, tomographic data have been used to extract other relevant complimentary information. In particular, a simple method based on the analysis of X-ray radiographs has been used to obtain a quantitative estimate of the global X-ray linear attenuation coefficient $(\mu)$ of the investigated samples; the presented procedure aims at providing a fast but accurate tool to indirectly extract information about relevant parameters of cement pastes, such as their actual w/c ratio and the amount of micro- and nano-scale porosity. Not only the advantages but also the practical limitations of using $\mathrm{X}$-ray micro-tomography for the analysis of cement-based 
materials are illustrated. Some fundamental issues related to experimental conditions, sample preparation and image analysis, often not sufficiently taken into account, are addressed and discussed as well.

\section{Materials and methods}

Sample preparation

A CEM I 52.5 R (EN 197-1 classification) ordinary Portland cement (OPC) was used for the preparation of the samples investigated in this study. The selected cement included a $5 \%$ by mass of Ca sulphates (gypsum, bassanite, anhydrite) acting as set retarders. The phase composition of the dry cement, determined by means of the Rietveld quantitative phase analysis (QPA) based on X-ray powder diffraction (XRPD) data is reported in Table 1. The presence of a small amount of portlandite (calcium hydroxide) in the dry mix can be related to the presence of free lime in the clinker or to the partial hydration of $\mathrm{Ca}$ silicates exposed to air moisture. The concentrations of major and trace elements in the same sample, obtained by means of an X-ray fluorescence (XRF) analysis are shown in Table 2.

Two cement pastes (Samples A and B) were prepared based on different formulations, corresponding to standard and well-characterized mixes commonly used in practical applications. Sample A was obtained by mixing the reference cement with de-ionized water, at a water-to-cement mass ratio $(\mathrm{w} / \mathrm{c})$ of 0.5 . Sample $\mathrm{B}$ was obtained by hydrating the same cement in the presence of a polycarboxylate ether superplasticizer (PCE-SP). PCE superplasticizers are essential constituents in the formulation of modern concretes; they are added in order to reduce the water content in a mixture, preserving at the same time a good workability but they are also employed to produce

Table 1 Phase composition of the selected OPC (clinker plus sulphates) determined by means of Rietveld QPA; a PANalytical X'Pert PRO MPD diffractometer (Bragg-Brentano geometry) equipped with a PIXcel detector was used for XRPD data collection

\begin{tabular}{|c|c|c|c|}
\hline Phase & Chemical formula & Cement notation & Weight $\%$ \\
\hline Alite & $\mathrm{Ca}_{3} \mathrm{SiO}_{5}$ & $\mathrm{C}_{3} \mathrm{~S}$ & $58.3(4)$ \\
\hline Belite & $\mathrm{Ca}_{2} \mathrm{SiO}_{4}$ & $\mathrm{C}_{2} \mathrm{~S}$ & $19.6(3)$ \\
\hline Aluminate & $\mathrm{Ca}_{3} \mathrm{Al}_{2} \mathrm{O}_{6}$ & $\mathrm{C}_{3} \mathrm{~A}$ & $7.3(2)$ \\
\hline Ferrite & $\mathrm{Ca}_{4} \mathrm{Al}_{2} \mathrm{Fe}_{2} \mathrm{O}_{10}$ & $\mathrm{C}_{4} \mathrm{AF}$ & $5.6(2)$ \\
\hline Periclase & $\mathrm{MgO}$ & M & $1.6(1)$ \\
\hline Gypsum & $\mathrm{CaSO}_{4} \cdot 2 \mathrm{H}_{2} \mathrm{O}$ & $\mathrm{CS} \mathrm{H}_{2}$ & $0.5(1)$ \\
\hline Bassanite & $\mathrm{CaSO}_{4} \cdot 0.5 \mathrm{H}_{2} \mathrm{O}$ & $\mathrm{CS} \mathrm{H}_{0.5}$ & $4.0(2)$ \\
\hline Arcanite & $\mathrm{K}_{2} \mathrm{SO}_{4}$ & $\mathrm{KS}$ & $1.2(2)$ \\
\hline Portlandite & $\mathrm{Ca}(\mathrm{OH})_{2}$ & $\mathrm{CH}$ & $1.7(2)$ \\
\hline
\end{tabular}

Table 2 Quantitative XRF chemical analysis of the selected cement carried out on a fused bead using a Philips PW 2400 sequential WDS spectrometer

\begin{tabular}{lrlllrlr}
\hline \multicolumn{2}{l}{ Major oxides (wt\%) } & \multicolumn{5}{c}{ Other elements (ppm) } \\
\hline $\mathrm{CaO}$ & 64.65 & $\mathrm{TiO}_{2}$ & 0.30 & $\mathrm{~S}$ & 23693 & $\mathrm{Zn}$ & 214 \\
$\mathrm{SiO}_{2}$ & 21.01 & $\mathrm{P}_{2} \mathrm{O}_{5}$ & 0.16 & $\mathrm{~V}$ & 155 & $\mathrm{Rb}$ & 42 \\
$\mathrm{Al}_{2} \mathrm{O}_{3}$ & 4.75 & $\mathrm{Na}_{2} \mathrm{O}$ & 0.13 & $\mathrm{Cr}$ & 92 & $\mathrm{Sr}$ & 1101 \\
$\mathrm{Fe}_{2} \mathrm{O}_{3}$ & 2.37 & $\mathrm{MnO}$ & 0.08 & $\mathrm{Co}$ & 7 & $\mathrm{Zr}$ & 54 \\
$\mathrm{MgO}$ & 1.93 & Total & 96.17 & $\mathrm{Ni}$ & 68 & $\mathrm{Ba}$ & 791 \\
$\mathrm{~K}_{2} \mathrm{O}$ & 0.79 & LOI & 1.66 & $\mathrm{Cu}$ & 175 & $\mathrm{~Pb}$ & 37 \\
\hline
\end{tabular}

LOI loss on ignition

high-flowing concretes. Their behaviour and effectiveness are governed by complex mechanisms of adsorption, electrosteric stabilization, deflocculation and dispersion of cement grains in the fresh mixture that is strictly related to the structural architecture of the different polymers. The selected superplasticizer was added to the mix of sample B at a dosage of $0.6 \%$ of the total mass. The w/c ratio was reduced to 0.37 in order to obtain a comparable rheological behaviour for the two mixtures, on the basis of mini-slump test results [18]. At the selected dosage of SP, a w/c of 0.37 produced the same measured flow (i.e. the same initial workability) of the cement paste with w/c of 0.5 in the absence of SP.

The cement powder (approximately $50 \mathrm{~g}$ ) and de-ionized water (including the superplasticizer for sample B) were mixed by hand for $1 \mathrm{~min}$ and then manually inserted into cylindrical borosilicate glass capillary tubes characterized by a low X-ray absorption. The internal diameter of the tubes was in the range of 400-600 $\mu \mathrm{m}$, with a wall thickness of $\sim 20 \mu \mathrm{m}$. The tubes were sealed with plasticine at both ends in order to prevent any loss of mixing water by evaporation. For each of the two samples, X-ray tomographic scans were acquired at increasing hydration ages, from a minimum of approximately $30 \mathrm{~min}$ after mixing, up to a maximum of 2-3 days. Particular care was taken to always ensure the scan of the same region for each sample, in order to obtain a consistent in-situ comparison of the cement paste evolution as a function of time. After preparation and between different tomographic scans, the samples were cured at room temperature in sealed glass vessels.

X-ray micro-tomography setup

X-ray micro-tomography experiments were carried out at the ID22 beamline [19] of the European Synchrotron Radiation Facility (ESRF) in Grenoble, France. The energy of the incident X-ray beam was set to $14 \mathrm{keV}$ $(\lambda=0.0886 \mathrm{~nm})$ using a double-crystal monochromator. The selected energy ensured a good balance in terms of 
$\mathrm{X}$-ray penetration and absorption contrast between different materials. Besides a more precise reconstruction of the variations of the linear attenuation coefficient within samples, the use of a monochromatic radiation (only applicable with synchrotron-based $\mathrm{X}-\mu \mathrm{CT}$ systems due to the high photon flux densities available) prevented any artefact related to the beam-hardening effect [4, 20-22]. Owing to the finite propagation distance of $24 \mathrm{~mm}$ between sample and detector, edge enhancement by means of inline (propagation-based) phase contrast contributed to the overall signal as well [23-25]. Phase contrast imaging is based on the fact that the refractive index for X-rays is slightly different from unity and an X-ray wave is modulated in its amplitude and phase after passing through a sample. The refractive index $n$ of a material for X-rays is conveniently described by a complex number consisting of a real and an imaginary part, usually written as $n=1-$ $\delta+i \beta$, where $\delta$ is the refractive index decrement (in the order of $10^{-5}-10^{-6}$ ), proportional to the electron density of the material, and $\beta$ (extinction coefficient) is responsible for the amount of attenuation of the wave and is related to the absorption coefficient $(\mu)$ and wavelength $(\lambda)$ by the equation $\mu=(4 \pi \beta) \cdot \lambda^{-1}$. Basically, when $\mathrm{X}$-rays pass through an object, a phase shift depending on $\delta$ occurs, leading to a deviation of the wavefronts. When using a sufficiently coherent radiation, as a result of local focusing and defocusing of the deviated X-rays, the formation of bright and dark fringes at the interfaces between different materials is observed, especially in the presence of high density gradients (e.g. at the sample/air interface). In other words, the interference at finite distances between mutually coherent parts of the beam that experienced different phase shifts is used to transform local phase variations into intensity variations recorded in the radiographs. This effect is enhanced by increasing the sample-detector distance, which is typically in the order of $\mathrm{cm}-\mathrm{dm}$, whereas a simple absorption image is obtained if the distance is very small (in the range of a few $\mathrm{mm}$ ).

For each tomographic scan, $1800 \mathrm{X}$-ray projections (Fig. 1) were acquired with an angular step of $0.1^{\circ}$ over a $180^{\circ}$ rotation. Raw projection data were collected using the ESRF-in-house developed FReLoN 2000 CCD camera [26], equipped with a $2048 \times 2048$ pixels chip with a pixel size of $14 \mu \mathrm{m}$. In this device, a CCD is optically coupled to a 3.5- $\mu \mathrm{m}$-thick Eu-doped $\mathrm{Lu}_{3} \mathrm{Al}_{5} \mathrm{O}_{12}$ (LAG:Eu) scintillator screen that converts the incoming $\mathrm{X}$-ray photons into visible light [27]. The optical system attached to the camera operated with a $\times 20$ magnification, resulting in a final pixel size of $0.7 \mu \mathrm{m}$. The exposure time was kept as low as possible in order to reduce the total duration of the experiments, which is a crucial aspect for the investigation of rapidly evolving cementitious systems, especially during the initial stages of hydration, when the rate of reaction is

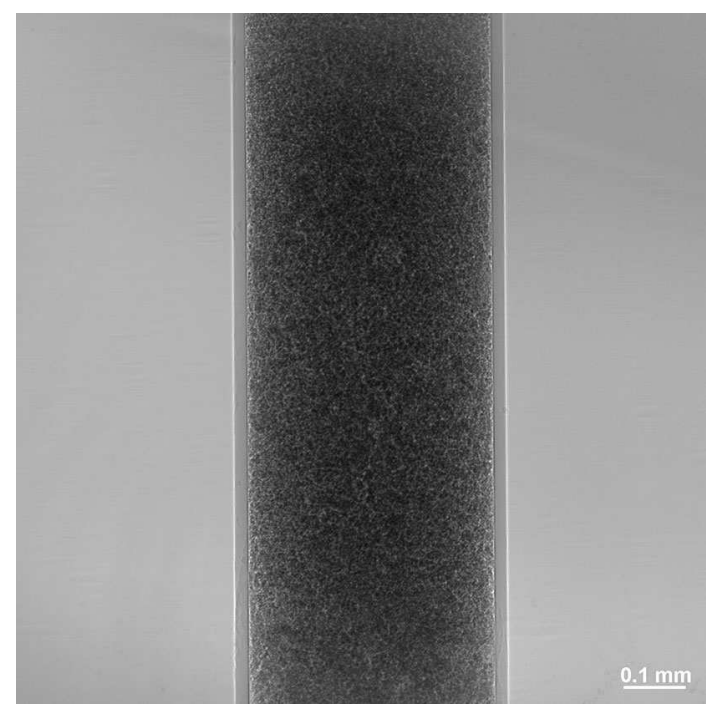

Fig. 1 X-ray radiograph (projection) of a glass capillary tube filled with an OPC paste

particularly fast. At the same time, the short exposures allowed to reduce the thermal load to the sample due to its interaction with the beam, minimizing water evaporation that may alter the nominal water-to-cement ratio of the mix and lead to undesired movements within the fresh paste. Small adjustments to the exposure time, which was in the range of $650-800 \mathrm{~ms}$ per projection, were necessary in order to compensate for the different densities and sizes of the samples and for the primary beam decay with time. By using the described setup, each scan took approximately 30-35 min (including multiple flat- and dark-field acquisitions), which proved to be an acceptable timescale for the in-situ study of the reactions taking place in the hydrating cement paste, except for the very early stages characterized by fast reactions. Three-dimensional stacks of cross-sectional slices (1600 contiguous images, corresponding to $\sim 1.1 \mathrm{~mm}$ in height) were reconstructed from projections data using the filtered back-projection algorithm [4] for parallel beam geometry implemented in the ESRF software package $P y H S T$ [28, 29].

\section{Image pre-processing}

The analysis of the 3D datasets was focused on the temporal evolution of the cement paste microstructure during the setting and hardening processes. In particular, the microstructural changes occurring within a volume of interest (VOI) were evaluated by analysing the same portion of each sample at different ages and comparing the variations of grey values in the tomographic images. Generally speaking, among the different crystalline and amorphous constituents of cementitious systems, anhydrous clinker phases exhibit the highest density and 
therefore the highest values of linear absorption coefficient $(\mu)$, whereas hydration products typically show a lower $\mathrm{X}$-ray attenuation. The values of $\mu$ for pure selected phases were calculated for an energy of $14 \mathrm{keV}$ using the X-ray database available in [30] and the phase densities reported in [31]. In Portland cements, calcium alumino ferrite $\left(\mathrm{C}_{4} \mathrm{AF}\right.$ in cement chemistry notation) shows by far the highest $\mu$ value $\left(109.20 \mathrm{~cm}^{-1}\right)$, followed by tricalcium silicate $\left(\mathrm{C}_{3} \mathrm{~S}, \quad 67.95 \mathrm{~cm}^{-1}\right)$, dicalcium silicate $\left(\mathrm{C}_{2} \mathrm{~S}\right.$, $\left.63.89 \mathrm{~cm}^{-1}\right)$ and tricalcium aluminate $\left(\mathrm{C}_{3} \mathrm{~A}, 56.34 \mathrm{~cm}^{-1}\right)$, respectively. For what concerns the hydration phases, the absorption coefficients vary from a maximum in portlandite (calcium hydroxide, $45.68 \mathrm{~cm}^{-1}$ ) to a minimum in ettringite $\left(17.00 \mathrm{~cm}^{-1}\right)$. The absorption coefficient of the most abundant hydration products, i.e. calcium-silicate hydrates $(\mathrm{C}-\mathrm{S}-\mathrm{H})$, lies between these two end members but can vary significantly depending on the density and content of structural and gel water, as well as on $\mathrm{C}-\mathrm{S}-\mathrm{H}$ nanoporosity. In practice, the discrimination of each single phase using X-ray micro-tomography remains an extremely difficult task, not only because of the small absorption variations between various crystalline and amorphous phases but also because of the extreme complexity of cement microstructure. The difference in $\mu$ between air and water is below the resolution of the adopted experimental configuration, hence no clear distinction can be made between entrapped air voids, self-desiccation pores and capillary water on the basis of the voxels grey values. In addition, the size of the features to be observed typically approaches the spatial resolution limit of the technique, thus further complications may arise from the fact that the volume corresponding to a single voxel may often be occupied by two or more different phases such as intergrown hydration products and nano-pores filled with air or water. Hence, the reconstruction process will attribute to each of these "mixed" voxels, a grey value that is proportional to the weighted mean of the attenuation coefficients of the phases contained in it (partial volume effect).

In order to obtain statistical and quantitative information about the evolution of the hydrating cement systems, histograms of the grey values distribution at different hydration ages were considered for each sample. Cylindrical volumes of interest (VOI) laterally delimited by the inner wall of the glass tubes were accurately selected at identical positions from each 3D dataset and their grey values histograms were then compared. The width of the VOIs ranged from $\sim 600$ to $\sim 850$ voxels $($ voxel size $=0.7 \mu \mathrm{m}$ ) depending on the diameter of the capillary tubes, while the vertical extent was limited to 120 slices in order to restrict the selection to portions of the paste characterized by high homogeneity. Larger VOIs were not considered, in order to exclude regions of the cement paste affected by large macropores, fractures and air bubbles caused by the inhomogeneous filling of the glass tubes. The total number of voxels considered in the VOIs ranged between $\sim 30 \times 10^{6}$ and $\sim 70 \times 10^{6}$; from a statistical point of view, the examined volumes can be reasonably considered representative of the phase ensemble.

Before calculating the grey values histograms, a $3 \times 3 \times 3$ median filter was applied in order to even out brightness and contrast variations between adjacent slices and reduce image noise. It has to be pointed out that, in order to perform reliable comparisons between images acquired with different scans, the mean greyscale values of each phase must be consistent throughout all the different datasets. However, owing to experimental issues (e.g. variations of the maximum and minimum absorption values within the sample, limitations of the tomographic reconstruction algorithms), this condition is usually not met. Therefore, brightness and contrast variations arising between different datasets had to be corrected prior to the calculation of histograms. For this kind of calibration, regions of interest within particular portions of the samples were defined and then compared for the various scans. In particular, the glass of the capillary tubes and the central portion of the largest clinker particles were chosen as reference materials because they can be assumed to remain unaltered during the hydration process (at least in the temporal scale investigated in this experiments). In general, a good correspondence was found between different datasets (typical differences in the mean value of reference regions were on the order of 1-2 grey values on an 8-bit scale), hence only minor adjustments in brightness and contrast were necessary. It should be pointed out that, owing to the particular experimental configuration adopted, the grey values in the reconstructed images are not only a function of the X-ray absorption contrast between different materials, but also depend on the effect of phase contrast [23-25] that is related to the sample-detector distance. Owing to X-ray refraction at interfaces within the specimen, phase contrast leads to the formation of bright and dark fringes in the recorded X-ray projections. As a result, edges appear significantly enhanced after reconstruction, with positive effects on the visualization of features (e.g. grain boundaries) and image interpretation, but at the same time the grey values in the slices become altered in proximity of the interfaces. A semi-quantitative estimate of the relative phase volumes can be achieved, therefore, only by the application of phase retrieval methods [32-34] that allow the reconstruction of maps of the electron density distribution within a sample, starting from projections acquired in phase contrast mode. Work is in progress to apply such methods to the quantification of phases present in cementitious systems. However, it should be pointed out that (1) fully quantitative phase retrieval in a single-distance manner is hardly possible nowadays unless a lot of a 
priori information about the sample is known; (2) multidistance phase retrieval is not possible due to dose and time constraints and (3) heterogeneous samples such as cement pastes are generally quite hard to handle for phase retrieval based on inline phase contrast images.

\section{Results and discussion}

Qualitative analysis of cement microstructure

Two slices from samples A and B, investigated approximately at the same hydration age, have been compared in Fig. 2. The relative scale of grey values in tomographic images of cement pastes is roughly similar to the one commonly observed in SEM-BSE imaging, although the physical process of image contrast generation is significantly different between the two techniques. Anhydrous clinker grains appear as bright particles (with a maximum size of approximately $50-60 \mu \mathrm{m}$ ), dispersed in a matrix of intergrown hydration phases (intermediate grey values), including also air- or water-filled porosity (dark areas). The clusters showing a slightly brighter grey value among the hydrated matrix correspond to large crystal aggregates of portlandite. The typical texture within clinker grains, characterized by $\mathrm{C}_{3} \mathrm{~S}$ and $\mathrm{C}_{2} \mathrm{~S}$ crystals embedded in a matrix of interstitial $\mathrm{C}_{3} \mathrm{~A}$ and $\mathrm{C}_{4} \mathrm{AF}$ can be sometimes recognized but not clearly resolved at the present level of spatial resolution. Large voids are caused by the inhomogeneous filling of the glass capillaries with the fresh cement paste. One interesting aspect that can be qualitatively evaluated in Fig. 2 is the different levels of packing of clinker grains in the two samples. As expected, the sample prepared with a w/c of 0.37 shows a significantly higher density of particles, as well as a more compact matrix.

In Fig. 3, the evolution of the microstructure of sample $\mathrm{A}(\mathrm{OPC}, \mathrm{w} / \mathrm{c}=0.5)$ from $1.75 \mathrm{~h}$ to $\sim 3$ days is illustrated using slices from different scans, corresponding closely to the same virtual section. The amount of deviation from perfect co-planarity, due to manual sample repositioning on the sample holder, has been estimated to be less than $0.5^{\circ}$. An initial attempt to scan a sample during the first tens of minutes after preparation gave poor results, as a consequence of small movements of the fresh paste and to the extremely rapid evolution of the microstructure during this stage. A decrease in the total scan duration is of course possible by reducing the number of projections and exposure time, although this would result in increased artefacts and reduced contrast in the reconstructed images; for such reasons, this possibility was not considered.

The effect of the hydration reactions is seen as a progressive dissolution of the original clinker grains (brighter particles) and the consequent growth of lower density hydration products (intermediate grey values) filling the pores initially occupied by water and air (darker grey values). At least two levels of attenuation may be identified within the hydrated portion of the sample. The less absorbing, which is also the most abundant, largely corresponds to $\mathrm{C}-\mathrm{S}-\mathrm{H}$ and is uniformly distributed throughout the sample around unreacted clinker particles; also ettringite and other minor phases appear in the images with a similar level of attenuation as $\mathrm{C}-\mathrm{S}-\mathrm{H}$, but they cannot be separated only on the basis of their mean grey value. Hydration rims around clinker particles are clearly observed, in particular, at higher hydration ages. The highest grey values among hydrated phases can be identified with portlandite, which typically grows in large and
Fig. 2 Two cross-sectional slices showing the effect of the water-to-cement ratio on the packing of clinker grains (brighter particles) for sample A (left) and B (right). The region outside the glass tube walls has been set to black in order to aid visualization

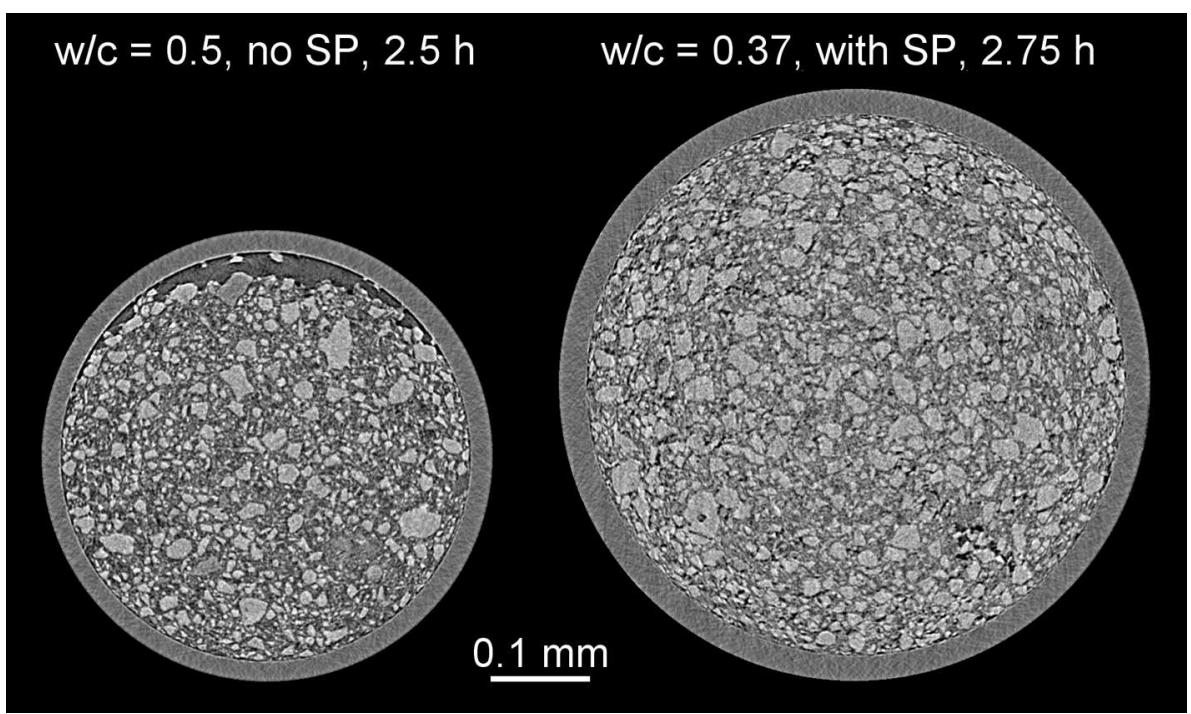




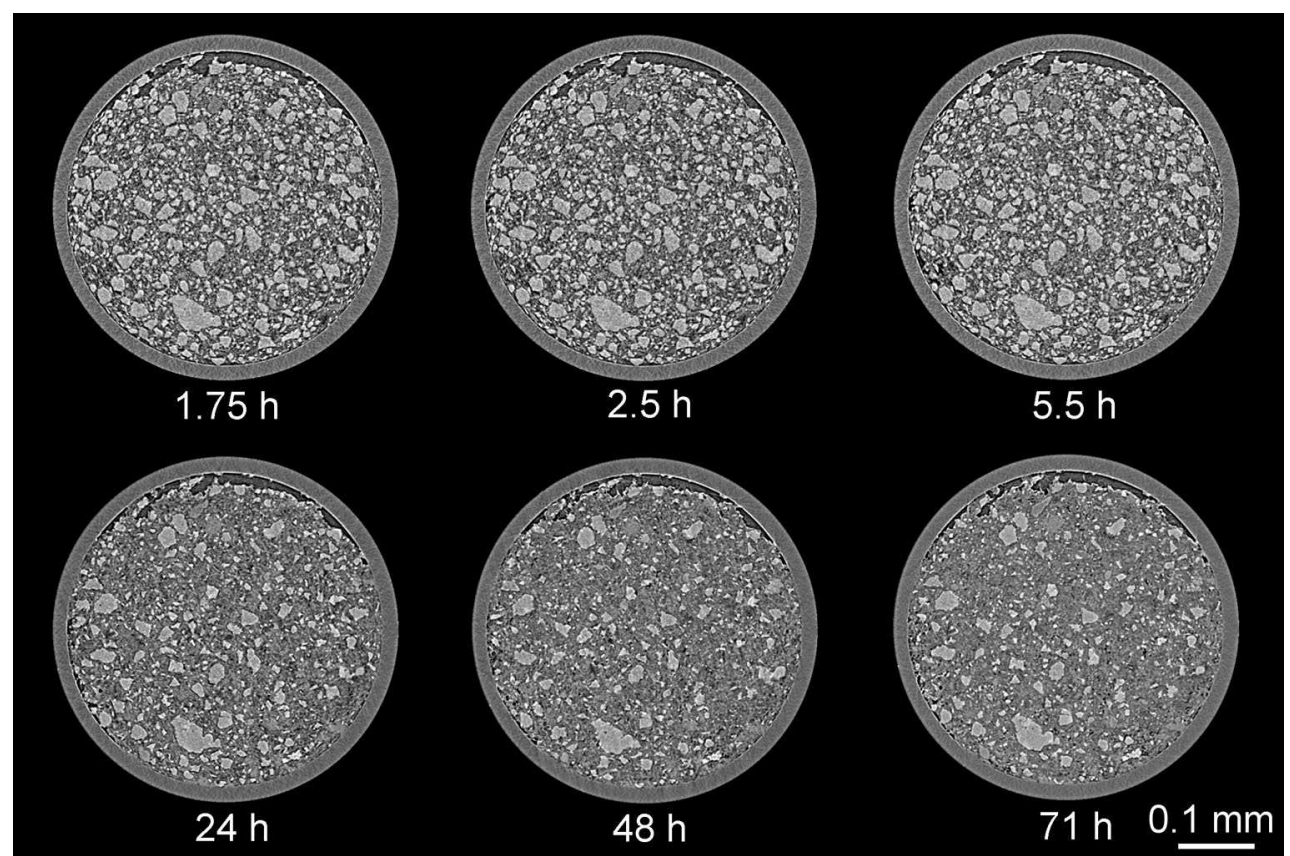

Fig. 3 Tomographic slices showing the evolution of the microstructure with hydration age in the same cross-sectional plane for sample A (w/ $\mathrm{c}=0.5$ ) at different stages of hydration. The region outside the glass tube walls has been set to black in order to aid visualization

Fig. 4 Segmented volume images showing the evolution of the unreacted cement fraction with time in a VOI of $100 \times 100 \times 100$ voxels $(70 \times 70 \times 70 \mu \mathrm{m})$ for sample A. As a result of hydration reactions, smaller clinker particles are rapidly consumed and larger grains (arrow) significantly reduce their size

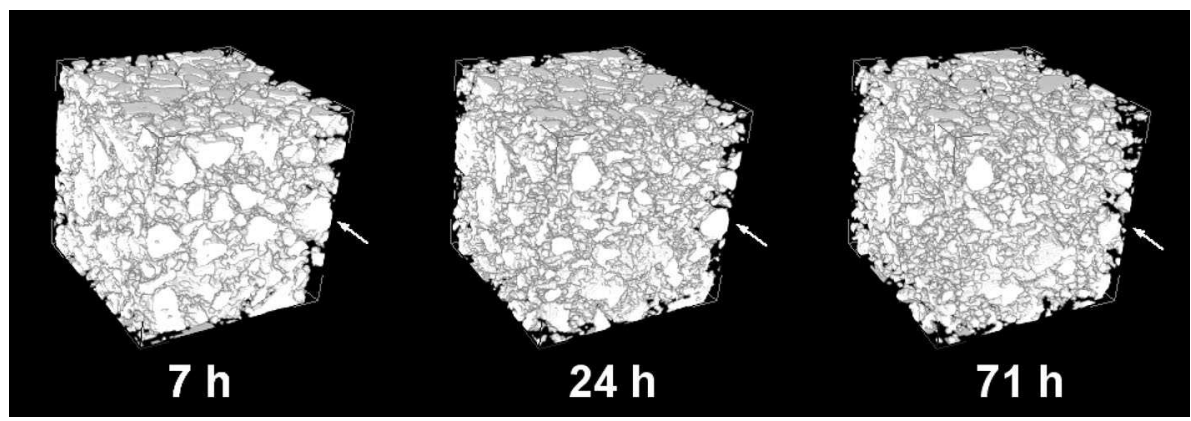

isolated platy crystals. The microstructural changes occurring within the cement paste can be monitored also by means of a 3D visualization as shown in Fig. 4. The unhydrated clinker fraction was segmented from a VOI of $70 \times 70 \times 70 \mu^{3}$ and then visualized by setting the remaining parts of the sample (pores and hydration products) to full transparency. By repeating the same operation on datasets acquired at different ages, the progressive reduction in size of clinker particles can be directly observed in 3D.

The microstructural evolution of the cement paste of sample A can be evaluated also in the graph of Fig. 5, where the grey values distribution at different hydration ages have been reported. In an ideal case (high density contrast and no mixed voxels), each phase would be characterized by a well-defined mean grey value and the histogram would be constituted of a number of sharp and isolated peaks. However, in real experimental data, peak

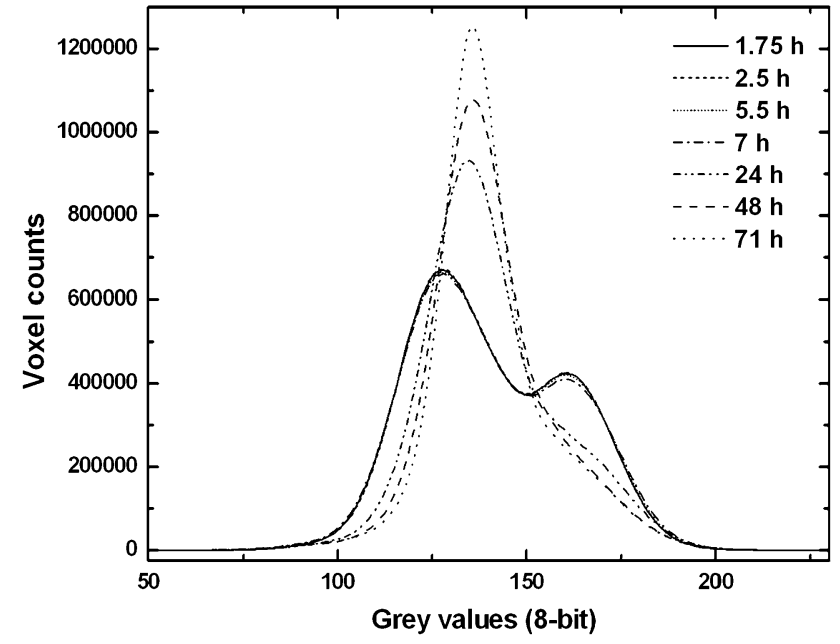

Fig. 5 Grey values distribution within a selected VOI at different hydration ages for sample A $(\mathrm{OPC}, \mathrm{w} / \mathrm{c}=0.5)$; an evolution from a bimodal to a unimodal distribution is clearly observed 
broadening occurs due to, e.g. (1) partial volume effect, (2) overlapping of grey value ranges relative to phases having a similar $\mu$, (3) the presence of image noise, (4) real density variations within a constituent and (5) edge enhancements due to inline X-ray phase contrast. In Fig. 5, at early hydration ages (up to $7 \mathrm{~h}$ ), the grey value curves show a bimodal distribution, where the first peak (lower grey values) largely corresponds to air- or water-filled porosity and early hydration products, while the second can be attributed to unreacted clinker particles. As the age of the sample increases (24, 48 and $71 \mathrm{~h}$ ), a significant change in the shape of the graph is observed. In particular, the evolution from a bimodal to a unimodal distribution is clear, with an intermediate peak growing at the expense of those relative to pores and anhydrous clinker. This variation reflects the progressive hydration of clinker, forming lower density hydrates (mainly $\mathrm{C}-\mathrm{S}-\mathrm{H}$ and portlandite) that fill the available porosity. A limited shift of the central peak towards higher grey values can be observed between 24 and $48 \mathrm{~h}$, as a consequence of the increased density of the hydration products in the cement matrix. In both the slices and the histograms, a major jump in the evolution of the microstructure occurs between 7 and $24 \mathrm{~h}$, with the rapid development of a considerable amount of hydration products. This can be interpreted as an effect of the increased reaction rate of $\mathrm{C}_{3} \mathrm{~S}$, corresponding to the beginning of the well-known acceleration period of hydrating Portland cements. On the contrary, almost overlapping trends of the histograms are observed from 1.75 to $7 \mathrm{~h}$, suggesting that the investigated cement was still within the induction (or dormant) period during these stages. Although the sequence of reactions is clear and seems to correspond to that observed in macroscopic systems by calorimetric techniques, the rate of reaction appears slowed down compared to real scale cement systems with a similar formulation. Such behaviour can be attributed in part to an inadequate initial mixing of the cement but another important aspect that may have played a role in the retardation of the reactions is the low amount and dispersion of the self-generated heat of hydration, as a consequence of the very small size of the glass vials. The problem is of course common to other experimental techniques that adopt the same kind of sample preparation for the study of cements (e.g. in-situ powder diffraction) and should be carefully taken into account for future experiments. The use of in-situ heating devices for temperature control around the sample may represent an option to overcome these inconveniences but such methods typically add serious limitations to the experimental setup of $\mathrm{X}-\mu \mathrm{CT}$.

The same procedure described for sample $\mathrm{A}$ was followed also for sample B (OPC, w/c $=0.37$ with the addition of a PCE superplasticizer), although a smaller number of scans were performed on this sample, reaching a maximum hydration age of $\sim 2$ days. Cross-sectional slices showing the microstructural evolution as a function of hydration age are reported in Fig. 6, while the variations of the grey values distribution are shown in Fig. 7. If compared to the previous sample, a lower degree of modification can be observed within the microstructure of the paste, indicating in general a slower evolution trend. The dissolution of clinker particles and the filling of pores are not easily recognizable because the largest cement grains and pores do not show a significant reduction in size. In addition, the global shape of the grey value curves appears to be considerably different compared to sample A, showing in this case an initial less defined separation (and a lower difference in height) between the two peaks of the bimodal distribution. This latter aspect is related to the different water-to-cement ratios of the two samples and in particular to the lower amount of pores and higher packing of cement particles in sample B. Furthermore, the deflocculation of clinker particles caused by the superplasticizer may have contributed to increase the interfacial surface. The combination of these effects results in a higher probability of sampling volumes containing phases with a marked difference in the value of the absorption coefficient (partial volume effect). However, the overall evolution trend is similar to that already observed for sample A, although less evident, with a progressive increase of the hydration products peak (that also shifts towards higher grey values) at the expenses of unreacted clinker. The rate of reaction appears further slowed down compared to the previous sample, as a consequence of the much lower water content of the cement mix. A possible retarding effect on the hydration caused by the superplasticizer may also be present, although the dominant effect is the large difference in w/c between the two samples.

\section{Quantitative estimate of the relative phase volumes}

Phase segmentation of tomographic images relative to cement paste microstructures has been attempted in previous studies and, although some quantitative information on the microstructural development and time evolution of the phase proportions can be obtained, the procedure suffers from issues related to spatial resolution. Even though excellent sub-micron resolution can be achieved by the use of synchrotron sources and proper experimental setups, the intrinsic length scale of cement features such as nanoporosity and nano-scale $\mathrm{C}-\mathrm{S}-\mathrm{H}$ colloidal aggregates prevents the whole spectrum of microstructural details to be properly resolved. In a previous work, Gallucci et al. [10] clearly showed that the measured porosity content and pore connectivity are strongly affected by the voxel size and in particular that the segmented porosity decreases with increasing voxel size. Therefore, particular care has to be 
Fig. 6 Tomographic slices showing the internal microstructure of sample B $(\mathrm{w} / \mathrm{c}=0.37$, with superplasticizer) at different ages from preparation; the region outside the glass tube walls has been set to black in order to aid visualization

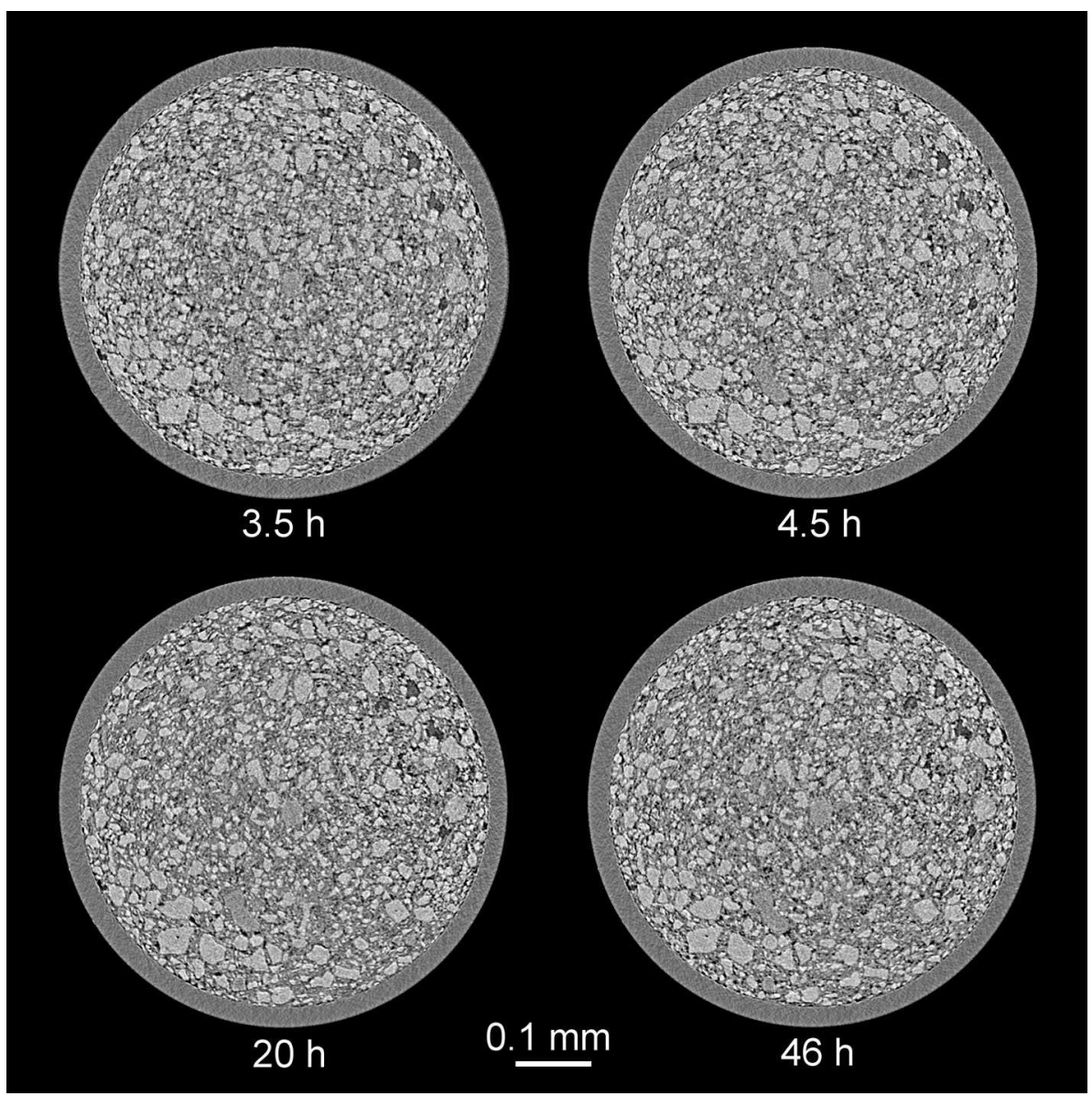

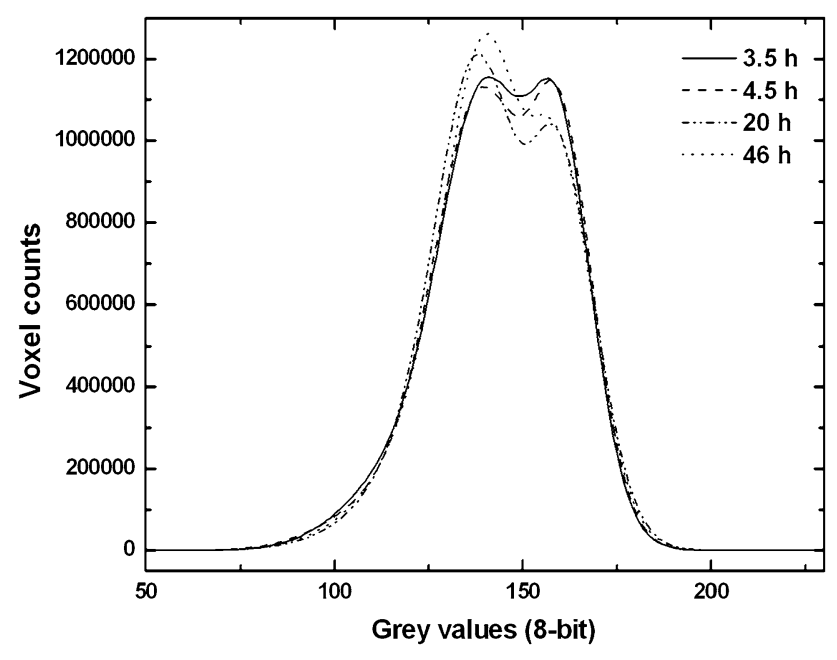

Fig. 7 Evolution of the grey values distributions for sample B (w/ $\mathrm{c}=0.37$, with superplasticizer) with hydration age; a different shape of the curves is observed if compared to sample A, owing to the much lower $\mathrm{w} / \mathrm{c}$

taken when tomographic images are used for quantitative analysis of cement microstructures. In this study, two different thresholds were applied to the grey values histograms of samples A and B in order to discriminate, as a function of hydration age, a) the unhydrated clinker grains and b) the air- or water-filled porosity. Owing to the high degree of overlap between the grey values shown by different phases, the determination of the correct threshold values for image segmentation is a challenging task and represents the main potential source of uncertainty. Even though some rigorous methods based on the histogram shape exist for the determination of thresholds, the best separation between different image features was obtained by manually selecting the appropriate values. Therefore, the thresholds were chosen on the basis of the best match between the borders of the segmented region and those of the known features in the images. The volume fractions of anhydrous cement and porosity were then calculated from the binarized dataset based on a simple cubic voxel model and the results are reported in Fig. 8. On the one hand, the clinker fraction can be reasonably segmented without any significant loss of data, being clinker particles characterized by a grain size that is approximately one to two orders of magnitude larger than the voxel resolution. On the other hand, the quantification of pores by image segmentation is highly underestimated because in cementitious systems the 

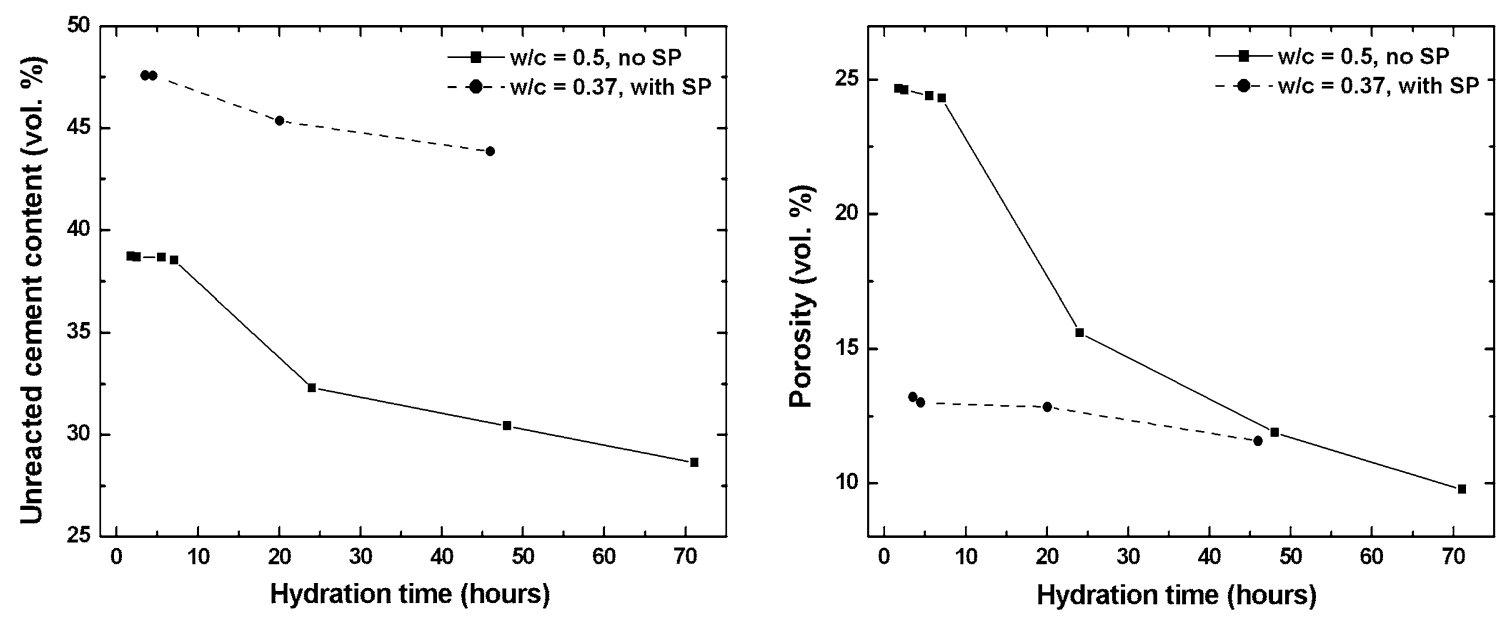

Fig. 8 Temporal evolution of the unreacted cement content (left) and pores (right) in sample A (solid line/squares) and B (dashed line/circles). Owing to the lower amount of water, a reduced initial porosity and a larger fraction of unreacted cement are observed in sample B

size of a significant fraction of pores is well below the voxel size adopted in this work. This is evident from the graphs of Fig. 8, where the amount of measured porosity extrapolated at $t=0$ (i.e. before mixing) is well below the actual volume fraction of water initially present in cement mixtures of the selected w/c ratios, thus leading to an overestimation of the hydration products. In particular, assuming a mean clinker density of $3.2 \mathrm{~g} / \mathrm{cm}^{3}$ and converting the mass ratios to volume ratios, the total initial (i.e. $t=0$ ) volume fraction of water-filled space (without considering entrapped air) in two cement pastes with w/c of 0.5 and 0.37 by mass should be equal to 61.6 and $54.3 \%$, respectively. Hence, it was assumed that the segmented porosity was constituted only by pores larger than the voxel size $(0.7 \mu \mathrm{m})$. The remaining, unsegmented voxels are constituted by a mixture of hydration products and smallscale capillary pores $(<0.7 \mu \mathrm{m})$. Furthermore, owing to the effect of phase contrast, pores and other features may be better detectable but their size may be significantly modified as a consequence of the presence of wide phase contrast interference fringes in the images (e.g. [35]).

Estimate of the mean absorption coefficient of cement pastes

The absorption of X-rays as a consequence of their interaction with matter is well described by the Beer-Lambert's law that relates the incident $\left(I_{0}\right)$ and the transmitted intensity $(I)$ to the X-ray linear attenuation coefficient $(\mu)$ according to the following equation:

$I=I_{0} \exp \left[-\sum_{i}\left(\mu_{i} x_{i}\right)\right]$,

where each increment $i$ represents a single phase with an attenuation coefficient $\mu_{i}$ and $x_{i}$ is the length travelled by
$\mathrm{X}$-rays through the $i$ th phase. For each single phase, $\mu$ is related to its density $(\rho)$, mean atomic number $(Z)$ and to the energy of the radiation $(E)$. In the energy range commonly used in X-ray imaging, the amount of attenuation is mainly determined by photoelectric absorption and Compton (inelastic) scattering.

In this work, in order to evaluate the actual absorption coefficient of the investigated cement pastes, a method based on the simple application of Beer-Lambert's law to radiographic images from micro-CT measurements was used. The results obtained were then compared with the calculated theoretical values in order to validate the adopted procedure. The knowledge of a mean value of $\mu$ for a cement paste is very important because the X-ray absorption coefficient is directly correlated to important properties such as w/c ratio, entrapped air content and phase composition, but can provide at the same time indirect information about microstructural features, such as nano-scale porosity, which cannot be detected by conventional imaging techniques. The applied method is based on the following simple assumption: if a certain propagation direction of a hypothetical point-like X-ray beam is considered and the thickness of the specimen along that trajectory is measured from a co-planar slice, the attenuation coefficient of the cement paste can be easily estimated by appropriately measuring the grey values on a radiograph perpendicular to the beam direction. In fact, it can be reasonably assumed that the grey values recorded in the normalized X-ray radiographs (i.e. the raw data divided by a flat field image), expressed as 32-bit floating point values, are equivalent to the $I / I_{0}$ ratio. Since the investigated cement pastes are usually enclosed in capillary tubes, the contribution of borosilicate glass walls $\left(\mu=14.38 \mathrm{~cm}^{-1}\right.$ at $14 \mathrm{keV}$, as calculated from the NIST database [36] ) to the total measured attenuation has to be taken into account. 
The measurement of the X-ray absorption of cement pastes from radiographs was carried out for samples A and B, considering all the datasets acquired at different hydration ages, under the assumption that the net effect of propagation-based phase contrast on the projection images can be neglected. Since flat field images were acquired with large angular steps, slight brightness variations were observed between different normalized radiographs as a consequence of the slow intensity decay of the primary beam with time; for this reason, radiographs were first rescaled in order to obtain a background value (i.e. beam propagating in air) of $I / I_{0}$ equal to 1 . For each sample, a section showing a high degree of homogeneity of the paste (i.e. with no macrovoids or air bubbles) was selected. The X-ray attenuation values $\left(I / I_{0}\right)$ were measured from radiographs perpendicular to the propagation direction, along two diametral chords lying on the plane of the selected slice, by measuring the mean grey value of a circle with a diameter of 15 pixels, centred on the path of the hypothetical X-ray point-like beam. In practice, the volume of sample considered for the measurement of the attenuation of X-rays corresponds approximately to a $10.5-\mu \mathrm{m}$-diameter cylinder. The choice of highly homogeneous regions of the sample is of course very important in order to obtain values of $\mu$ that reflects the actual mean X-ray absorption of the paste, minimizing the effects of the presence of macrovoids caused by the difficult filling of the capillary tubes. The analysis of the X-ray radiographs according to this method allowed obtaining an estimate of the overall attenuation coefficients of samples A and B at different hydration ages (Table 3), and such values are directly related to the w/c ratio, air content and average phase composition of the cement paste. Mean $\mu$ values of 26.52 and $29.61 \mathrm{~cm}^{-1}$ were obtained for sample A (w/c 0.5) and B (w/c 0.37),

Table 3 X-ray attenuation coefficients of the investigated cement pastes, as calculated from X-ray radiographs; for each scan, the reported value represents the average of two measurements carried out along different directions within portions of the samples characterized by high homogeneity and the absence of macropores

\begin{tabular}{lll}
\hline Sample & Hydration age $(\mathrm{h})$ & Attenuation coeff. $\left(\mathrm{cm}^{-1}\right)$ \\
\hline A w/c 0.5 & 1.75 & 26.49 \\
A w/c 0.5 & 2.5 & 26.42 \\
A w/c 0.5 & 5.5 & 26.32 \\
A w/c 0.5 & 7 & 26.61 \\
A w/c 0.5 & 24 & 26.53 \\
A w/c 0.5 & 48 & 26.65 \\
A w/c 0.5 & 71 & 26.61 \\
B w/c 0.37 & 3.5 & 29.58 \\
B w/c 0.37 & 4.5 & 29.79 \\
B w/c 0.37 & 20 & 29.44 \\
B w/c 0.37 & 46 & 29.63 \\
\hline
\end{tabular}

respectively. It can be observed that the absorption coefficient of the two cement pastes remains practically constant throughout the whole investigated temporal range, as a consequence of mass conservation in the sealed cement system. These results confirm the good reproducibility of the adopted procedure and indicate at the same time that no significant water losses by evaporation affected the samples.

In order to validate the obtained results, the theoretical mean attenuation coefficient of two cement pastes with a w/c of 0.5 and 0.37 was calculated, starting from the phase composition reported in Table 1 and using the attenuation coefficients available in [30]; a value of $\mu$ equal to $1.841 \mathrm{~cm}^{-1}$ was adopted for water at $14 \mathrm{keV}$. In this way, theoretical $\mu$ values of 26.78 and $31.52 \mathrm{~cm}^{-1}$ were obtained for sample A and B, respectively, assuming the absence of entrapped air. In general, considering all the potential sources of uncertainties, the theoretical values are in good accordance with those obtained from radiographs. In particular, for sample A the two values are very close, while for sample B a higher difference is observed. The measured values are always lower than the theoretical ones, as a consequence of air entrapment during the insertion of the fresh cement mix in the glass tubes, in particular for sample B. The filling operation is in fact very critical and may also lead to local alterations of the nominal w/c within the sample, even if carried out with the maximum care. In particular, previous experiences have indicated that introducing cement paste within the small glass vials has a tendency to lower their w/c ratio. It should be also pointed out that the theoretical absorption coefficients may be affected by the uncertainties in the cement composition that was used as a starting point for the calculations. In addition, the values of $\mu$ obtained from radiographs may suffer from uncertainties in the measurement of $I / I_{0}$ that should be taken into account as well.

\section{Conclusions}

The results presented in this work confirm the potentialities of synchrotron-based X-ray computed micro-tomography as a non-invasive tool for the investigation of the microstructural properties of cement-based materials. The access to real 3D information represents the major advantages of $\mathrm{X}-\mu \mathrm{CT}$ with respect to other imaging methods, allowing the investigation of the properties of cements over relatively large volumes, by far more representative than 2D surfaces. It was shown that SR $\mathrm{X}-\mu \mathrm{CT}$ is able to monitor small microstructural variations with an adequate time resolution, even though it was not possible to investigate with the same accuracy the first tens of minutes of reaction, mainly because of sample internal movements. The development of 
hydration products (mainly $\mathrm{C}-\mathrm{S}-\mathrm{H}$, portlandite and ettringite) at the expenses of anhydrous clinker grains and pores was visualized by comparing cross-sectional slices and 3D volumes extracted from datasets acquired at different hydration ages on the same sample, thus providing a real in-situ monitoring of the microstructural evolution. The amount of unreacted clinker and porosity as a function of hydration age was successfully quantified.

The spatial resolution of SR X- $\mu \mathrm{CT}$ is at present very good but still low compared to the scale of the features present in the microstructure of OPC pastes, which are characterized by the coexistence of a nano-scale porosity with several highly intermixed hydration phases showing similar attenuation coefficients. However, it has to be pointed out that no other experimental techniques are at present capable of imaging in 3D such a complex microstructure, with sub-micrometric resolution in a non-invasive manner.

Another technical limitation is represented by the limited size $(<1 \mathrm{~mm})$ of the samples that can be investigated if the maximum resolution has to be achieved. This aspect leaves some open questions about the degree of representativeness of real cementitious systems in practical applications. Furthermore, many issues related to sample preparation and experimental conditions (e.g. spatial resolution, scan duration, X-ray energy, sample size and mixing) have not been extensively discussed in the previous literature dealing with X-ray computed tomography on cementitious materials. All of these aspects are indeed very critical and may considerably affect the results of the experiments, especially in terms of kinetics of the reactions and therefore should be taken into account for future high resolution tomographic investigations of cements.

Further improvements in terms of spatial and temporal resolution will undoubtedly contribute in the future to make SR X- $\mu$ CT a unique tool for the in-situ investigation of complex and rapidly evolving systems. In particular, a reduction of the acquisition times will give the opportunity to clarify the phenomena occurring during the first minutes of cement hydration that are at present still not accessible by this experimental technique. In a recent study [37], SR $\mathrm{X}-\mu \mathrm{CT}$ using polychromatic radiation with acquisition times in the range of seconds per scan has already been successfully applied to dry concrete specimens.

In the present work, a simple method based on the analysis of radiographs for the determination of the X-ray attenuation coefficient of cement pastes was also illustrated. The procedure, if carefully applied, provides a simple tool to evaluate the correspondence between the nominal and actual properties of cement pastes and similar materials inserted in small glass vials, providing information about the level of representativeness of the samples. In particular, the method may be used for cement samples to obtain a rapid evaluation of, e.g. the amount of entrapped air or the actual w/c ratio of the cement paste and to verify the presence of artefacts related to sample preparation.

Acknowledgements This work was supported by the European Synchrotron Radiation Facility (exp. MA-648, MA-1063). The experiments were performed in the frame of the research agreement between Mapei S.p.A. and the Department of Geosciences of the University of Padua. The authors would like to acknowledge Cyril Guilloud and Sylvain Laboure (ESRF) for their precious help during the experiments at ID22.

\section{References}

1. Scrivener KL (2004) Backscattered electron imaging of cementitious microstructures: understanding and quantification. Cem Concr Compos 26(8):935-945

2. Stutzman P (2004) Scanning electron microscopy imaging of hydraulic cement microstructure. Cem Concr Compos 26(8): 957-966

3. Walsh D, Otooni MA, Taylor ME Jr, Marcinkowski MJ (1974) Study of Portland cement fracture surfaces by scanning electron microscopy techniques. J Mater Sci 9:423-429. doi:10.1007/ BF00737842

4. Kak AC, Slaney M (1988) Principles of computerized tomographic imaging. IEEE Press, New York

5. Bentz DP, Quenard DA, Kunzel HM, Baruchel J, Peyrin F, Martys NS, Garboczi EJ (2000) Microstructure and transport properties of porous building materials. II: three-dimensional X-ray tomographic studies. Mater Struct 33:147-153

6. Landis EN, Petrell AL, Lu S, Nagy EN (2000) Examination of pore structure using three-dimensional image analysis of microtomographic data. Concr Sci Eng 2:162-169

7. Bentz DP, Mizell S, Satterfield S, Devaney J, George W, Ketcham P, Graham J, Porterfield J, Quenard D, Vallee F, Sallee H, Boller E, Baruchel J (2002) The visible cement data set. J Res Natl Inst Stand Technol 107:137-148

8. Helfen L, Dehn F, Mikulík P, Baumbach T (2005) Threedimensional imaging of cement microstructure evolution during hydration. Adv Cem Res 17(3):103-111

9. Burlion N, Bernard D, Chen D (2006) X-ray microtomography: application to microstructure analysis of a cementitious material during leaching process. Cem Concr Res 36:346-357

10. Gallucci E, Scrivener K, Groso A, Stampanoni M, Margaritondo $G$ (2007) 3D experimental investigation of the microstructure of cements pastes using synchrotron X-ray microtomography $(\mu \mathrm{CT})$. Cem Concr Res 37:360-368

11. Promentilla MAB, Sugiyama T, Hitomi T, Takeda N (2009) Quantification of tortuosity in hardened cement pastes using synchrotron-based X-ray computed microtomography. Cem Concr Res 39(6):548-557

12. Gastaldi D, Canonico F, Capelli L, Boccaleri E, Milanesio M, Palin L, Croce G, Marone F, Mader K, Stampanoni M (2012) In situ tomographic investigation on the early hydration behaviors of cementing systems. Constr Build Mat 29:284-290

13. Stock SR, De Carlo F, Almer JD (2008) High energy X-ray scattering tomography applied to bone. J Struct Biol 161:144-150

14. Bleuet P, Welcomme E, Dooryhée E, Susini J, Hodeau J-L, Walter P (2008) Probing the structure of heterogeneous diluted materials by diffraction tomography. Nat Mater 7:468-472

15. Artioli G, Cerulli T, Cruciani G, Dalconi MC, Ferrari G, Parisatto M, Rack A, Tucoulou R (2010) X-ray diffraction microtomography 
(XRD-CT), a novel tool for non-invasive mapping of phase development in cement materials. Anal Bioanal Chem 397(6): 2131-2136

16. Valentini L, Dalconi MC, Parisatto M, Cruciani G, Artioli G (2011) Towards three-dimensional quantitative reconstruction of cement microstructure by X-ray diffraction microtomography. J Appl Crystallogr 44(2):272-280

17. Voltolini M, Dalconi MC, Artioli G, Parisatto M, Valentini L, Russo V, Bonnin A, Tucoulou R (2013) Understanding cement hydration at the microscale: new opportunities from "pencilbeam' synchrotron X-ray diffraction tomography. J Appl Crystallogr 46(1):142-152

18. Kantro DL (1980) Influence of water-reducing admixtures on properties of cement paste-a miniature slump test. Cem Concr Aggreg 2:95-102

19. Martinez-Criado G, Tucoulou R, Cloetens P, Bleuet P, Bohic S, Cauzid J, Kieffer I, Kosior E, Laboure S, Petitgirard S, Rack A, Angel Sans J, Segura-Ruiz J, Suhonen H, Susini J, Villanova J (2012) Status of the hard X-ray microprobe beamline ID22 of the European Synchrotron Radiation Facility. J Synchrotron Radiat 19(1):10-18

20. Jennings RJ (1988) A method for comparing beam-hardening filter materials for diagnostic radiology. Med Phys 15:588-599

21. Haibel A (2008) Synchrotron X-ray absorption tomography. In: Banhart $\mathrm{J}$ (ed) Advanced tomographic methods in materials research and engineering. Oxford University Press, New York, pp 141-160

22. Brooks RA, Di Chiro G (1976) Beam hardening in X-ray reconstructive tomography. Phys Med Biol 21:390-398

23. Snigirev A, Snigireva I, Kohn V, Kuznetsov S, Schelokov I (1995) On the possibilities of X-ray phase contrast microimaging by coherent high-energy synchrotron radiation. Rev Sci Instrum 66:5486-5492

24. Raven C, Snigirev A, Snigireva I, Spanne P, Souvorov A, Kohn V (1996) Phase-contrast microtomography with coherent highenergy synchrotron X-rays. Appl Phys Lett 69:1826-1828

25. Cloetens P, Ludwig W, Baruchel J, Guigay J-P, RejmankovaPernot P, Salomé-Pateyron M, Schlenker M, Buffière JY, Maire E, Peix G (1999) Hard X-ray phase imaging using simple propagation of a coherent synchrotron radiation beam. J Phys D 32:A145-A151

26. Labiche J-C, Mathon O, Pascarelli S, Newton MA, Guilera Ferre G, Curfs C, Vaughan G, Homs A, Fernandez Carreiras D (2007) The fast readout low noise camera as a versatile $\mathrm{X}$-ray detector for time resolved dispersive extended X-ray absorption fine structure and diffraction studies of dynamic problems in materials science, chemistry, and catalysis. Rev Sci Instrum 78:091301

27. Koch A, Peyrin F, Heurtier P, Ferrand B, Chambaz B, Ludwig W, Couchaud M (1999) X-ray camera for computed microtomography of biological samples with micrometer resolution using $\mathrm{Lu}_{3} \mathrm{Al}_{5} \mathrm{O}_{12}$ and $\mathrm{Y}_{3} \mathrm{Al}_{5} \mathrm{O}_{12}$ scintillators. SPIE Conference on Physics of Medical Imaging, San Diego 3659:170-179

28. Mirone A, Wilcke R, Hammersley A, Ferrero C (2012) PyHST (High Speed Tomographic Reconstruction), http://www.esrf.eu/ UsersAndScience/Experiments/TBS/SciSoft/

29. Mirone A, Brun E, Gouillart E, Tafforeau P, Kieffer J (2014) The PyHST2 hybrid distributed code for high speed tomographic reconstruction with iterative reconstruction and a priori knowledge capabilities. Nucl Instr Meth Phys Res B 324:41-48

30. Henke BL, Gullikson EM, Davis JC (1993) X-ray interactions: photoabsorption, scattering, transmission, and reflection at $\mathrm{E}=50$ $30000 \mathrm{eV}, \mathrm{Z}=1-92$, At Data Nucl Data Tables 54(2):181-342 (http://henke.lbl.gov/optical_constants/)

31. Bentz DP, Coveney PV, Garboczi EJ, Kleyn MF, Stutzman PE (1994) Cellular automaton simulations of cement hydration and microstructure development. Model Simul Mater Sci Eng 2: 783-808

32. Cloetens P, Ludwig W, Baruchel J, Van Dyck D, Van Landuyt J, Guigay J-P, Schlenker M (1999) Holotomography: quantitative phase tomography with micrometer resolution using hard synchrotron radiation X-rays. Appl Phys Lett 75:2912-2914

33. Weitkamp T, Haas D, Wegrzynek D, Rack A (2011) ANKAphase: software for single-distance phase-retrieval from inline X-ray phase contrast radiographs. J Synchrotron Radiat 18:617-629

34. Langer M, Cloetens P, Guigay J-P, Peyrin F (2008) Quantitative comparison of direct phase retrieval algorithms in in-line phase tomography. Med Phys 35(10):4556-4566

35. Zabler S, Riesemeier H, Fratzl P, Zaslansky P (2006) Fresnelpropagated imaging for the study of human tooth dentin by partially coherent X-ray tomography. Opt Expr 14(19):8584-8597

36. Hubbell JH, Seltzer SM (1996) Tables of X-ray mass attenuation coefficients and mass energy-absorption coefficients from $1 \mathrm{keV}$ to $20 \mathrm{MeV}$ for elements $\mathrm{Z}=1$ to 92 and 48 additional substances of dosimetric interest, http://www.nist.gov/pml/data/xray coef/index.cfm

37. Rack A, Garcia-Moreno F, Schmitt C, Betz O, Cecilia A, Ershov A, Rack T, Banhart J, Zabler S (2010) On the possibilities of hard X-ray imaging with high spatio-temporal resolution using polychromatic synchrotron radiation. J X-ray Sci Technol 18(4):429-441 